\title{
Environment-induced dephasing versus von Neumann measurements in proton tunneling
}

\author{
A. D. Godbeer, ${ }^{*}$ J. S. Al-Khalili, ${ }^{\dagger}$ and P. D. Stevenson ${ }^{\ddagger}$ \\ University of Surrey, Guildford, Surrey GU2 7XH, United Kingdom
}

(Received 26 January 2014; published 2 July 2014)

\begin{abstract}
In this work we compare two theoretical approaches to modeling the action of the environment on an open quantum system. It is often assumed that as the temperature of the environment surrounding a quantum system increases, so does the speed of environment-induced dephasing, or decoherence (dynamical noise), and so the efficacy of processes such as quantum tunneling drops. An alternative way of viewing the action of the environment is to consider it as carrying out von Neumann-type measurements that, in the limit of continuous observation, lead to the so-called quantum Zeno effect, whereby the system is never allowed to evolve because its wave function is collapsed to its initial state with overwhelming likelihood. However, it has been established in recent years that under certain conditions quantum processes such as tunneling can actually be enhanced (thermally assisted) when the system couples to its environment, as this allows transitions to higher-energy eigenstates closer to the top of the potential barrier. Here we show that, over a specific temperature range, increasing the temperature of the heat bath to encourage such thermally induced tunneling is equivalent to increasing the frequency of a von Neumann-type measurement on the system by the environment (an anti-Zeno effect). However, this correspondence between these two independent pictures of quantum measurement breaks down above a certain limit: Increasing the frequency of measurement above this limit leads to a reversal from an anti-Zeno to a Zeno effect and the tunneling rate decreases again, whereas raising the temperature further leads to a leveling off in the tunneling probability.
\end{abstract}

DOI: 10.1103/PhysRevA.90.012102

PACS number(s): 03.65.Xp, 03.65.Ta

\section{INTRODUCTION}

There are many examples in physics, chemistry, and, more recently, even biology of open quantum systems in which the relevant microscopic mechanisms or processes behave quantum mechanically, but which cannot be treated in isolation from their surrounding environment. Such systems are often embedded within complex molecular structures or are surrounded by water molecules. This external environment is often modeled as a heat bath of harmonic oscillators to describe thermal fluctuations arising from, for example, molecular vibrations. In such open quantum systems, coupling to the environment leads to the loss of quantum coherence at time scales that depend on the temperature of the heat bath and the strength of the coupling [1,2]. Many different terms are used to describe this process, such as relaxation, dissipation, dephasing, and decoherence. Open quantum systems are these days a subject of detailed study in situations where the coupling of the quantum system to its environment is a crucial feature of the phenomenon or mechanism of interest, such as in nuclear magnetic resonance. However, by and large, many of the best-studied features of the atomic and subatomic world are still dealt with as idealized isolated quantum systems.

In this paper we focus on one mechanism in particular: quantum tunneling. There are many examples in physics and chemistry where quantum tunneling only plays a role when the systems involved are at low temperatures or when the tunneling either involves low mass particles, such as electrons, or takes place over short, atomic, distances.

A well-studied example of proton tunneling in chemistry has been the double-H-bonded benzoic acid dimer [3-13],

\footnotetext{
*a.godbeer@surrey.ac.uk

†j.al-khalili@ surrey.ac.uk

${ }^{\ddagger}$ p.stevenson@ surrey.ac.uk
}

making this simple structure useful for modeling more complex chemical and biological systems that may involve proton tunneling. The first experimental evidence for proton tunneling in biological systems came in fact from the study of enzyme catalysis in 1989 (for the enzyme alcohol dehydrogenase, which transfers a proton from an alcohol molecule to a molecule of nicotinamide adenine dinucleotide), where the effects of atomic mass on reaction rates through isotopic substitution revealed clear evidence of quantum tunneling even at relatively high temperatures [14]. Since then, many other enzymatic reactions have been ascribed to proton tunneling and it has been established that, at low temperatures, proton tunneling dominates the proton-transfer dynamics [15-18].

It is now well established that in a number of biochemical processes there exists a subtle interplay between quantum coherence and environmental noise such that the action of the latter can assist rather than hinder the former. In this paper we examine a model of proton tunneling in a double-well potential under the influence of an external environment. We consider the link between quantum measurement and decoherence using numerical simulations such as has been described by several authors $[19,20]$, who consider a particle that starts off on one side of a double-well potential and investigate the effects of measurement on the time it takes for the particle to tunnel between the wells. However, in those studies conflicting conclusions are reached as to whether continuous measurement slows down the tunneling process [the quantum Zeno effect (QZE)] or speeds it up [the anti-Zeno effect (AZE)] [21]. For instance, the standard argument is that repeated measurement continually collapses the state of the particle with overwhelming likelihood back to its initial state on one side of the barrier. On the other hand, it is acknowledged that the act of measurement can excite the particle to higher energies and thus enhance the probability of barrier penetration. However, these models are relatively complex and make it difficult to 
see what is happening in a transparent way. The review by Koshino and Shimizu [22] provides a survey of the field, but also highlights the complexity of the problem to the extent that the physics can only be appreciated fully by the aficionados.

In particular, a model proposed by Kofman and Kurizki [23] is useful for highlighting the advantages of our approach. Their model deals with energy measurements associated with the decay of an unstable state. They derive a universal result that they claim shows that the anti-Zeno effect of accelerated decay is much easier to achieve than the Zeno effect itself, the latter being restricted to a limited class of systems due to a competition between the frequency of measurement and the energy spread brought about by this measurement due to the uncertainty principle. Moreover, as the energy uncertainty grows with the frequency of the measurements, the state is able to decay into a larger number of channels, thus accelerating the decay process. Their universal relation involves the convolution of two distributions: the measurement-induced level width and spectrum of energies to which the decaying state can couple.

The above model was extended in the work by Ruseckas and Kaulakys [24,25], who took into account both the finite duration and finite accuracy of the measurement. They showed that in fact both the QZE and the AZE can be realized depending on the properties of the system and the strength and frequency of the interaction. Just as in Kofman and Kurizki's work, this model also relies on the convolution of the two distributions. When the width of the spectral line (containing the physics of the interaction) is much broader than the width of the reservoir (the range of energy eigenstates available for the decay) the overlap between the two is small and the decay is inhibited (QZE). On the other hand, if the spectral line is narrow then more frequent measurements can broaden it and enhance its overlap with the reservoir spectrum, thus accelerating the decay.

The purpose of the work described in this paper is to compare the above lines of reasoning with the more traditional approach of dealing with dissipation in an open quantum system using a reduced density matrix model. Here we compare two very different ways of modeling the measurement problem. The first simulates a von Neumann-type process of irreversible reduction [26] via a position (Pointer state) measurement that entangles the state of the system with that of the measuring device (in this case the surrounding environment) causing a decay of the off-diagonal elements of the density matrix. The second involves adding a dissipative (Lindblad) term in the master equation for the time-dependent density matrix in order to model the (continuous) coupling of the system to a surrounding heat bath of oscillators. We refer to these two approaches as the Pointer and Lindblad methods, respectively. The latter method in particular is well known and has been studied extensively for various systems [27-44]; the comparison between the two methods and the possibility of opening up an alternative way of looking at the system-environment interaction are the primary goals of this paper, in addition to the hunt for the AZE, which may be necessary for the warm, wet environments found in biological organisms to exhibit quantum behavior.

In both approaches, our quantum system consists of a single proton in an asymmetric double-well potential, as found in many biological systems. The one-dimensional (1D) potential well we use is defined as a quartic function of position (defined in Sec. III) with parameters chosen to describe a benzoic acid dimer molecule in a crystal field. The two minima along the energy surface for a single benzoic acid dimer are highly symmetric, but introducing a crystal field causes asymmetry, which had previously been determined from temperaturedependent infrared absorptions and NMR data, making one state more energetically favorable than the other [5]. While our simple parametrization gives a good approximation of the behavior of the physical system, it is worth stating that our interest is to ultimately apply these techniques to study proton tunneling in biological systems, such as in enzyme catalysis and in hydrogen bonds between DNA base pairs, which feature similar asymmetric well potentials. That work will involve generating a more realistic double-well potential using density-functional theory to take into account the complex structure of large biomolecules.

\section{THEORY}

\section{A. Pointer measurements}

Many authors have investigated the measurement process in quantum mechanics, which entangles the state of the system with that of the measuring device causing a decay of the offdiagonal elements of the system's density matrix. Wallace [45] chose a simple example: the evolution of a one-dimensional wave packet describing the motion of a free particle. He considered the time evolution of the density operator in momentum space and then Fourier transformed it back to configuration space before simulating the measurement process by setting all off-block-diagonal elements of the density matrix to zero. He concluded that the effects of repeated measurement can have nontrivial dynamical effects both on the rate of the spreading of the wave packet and on the rate of its center-of-mass motion, sometimes speeding it up and sometimes slowing it down. However, since he only dealt with free particles he could say nothing about the effects of the measurement on quantum tunneling.

Recently, we extended the idea of Wallace to the more interesting case of the tunneling of a wave packet through a square potential barrier [46] to investigate the more realistic example of particle decay, which is closer in spirit to the models of Refs. [19,20,47]. We describe this approach here and connect it with the more physically realistic reduced density matrix approach described in the next section.

We simulate the process of position measurement at any given time for a certain choice of position resolution by setting to zero the off-diagonal terms in the density matrix (expanded in a position space basis). Specifically, we initiate an imprecise measurement on the system to essentially determine which well the proton is in. As such, only the off-diagonal quarters of the density matrix are set to zero, as shown in Fig. 1. Unlike other approaches that deal with the coupling of the quantum system to its environment and have to take into account the number of states available for the decay and the overlap of this (reservoir) spectrum with the measurement induced level width, we do not need to consider the reservoir at all.

We begin by writing the matrix elements of the density operator $\hat{\rho}(t)=|\psi(t)\rangle\langle\psi(t)|$ in 1D coordinate space 


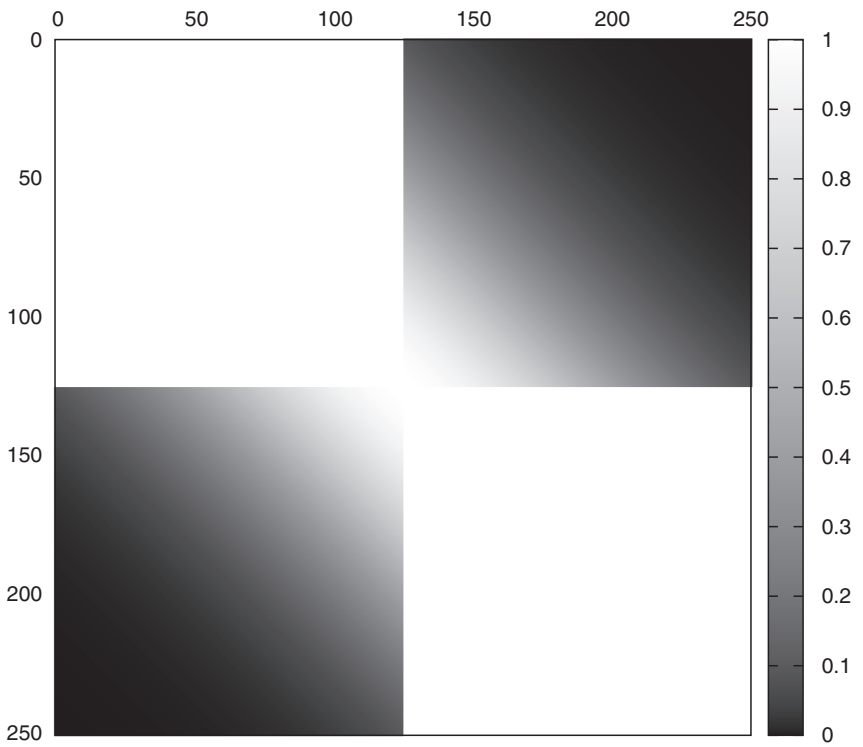

FIG. 1. Density matrix just after measurement showing how the off-diagonal elements in a block of size $N / 2$ are removed. White indicates regions of the matrix in which the elements are unchanged and black indicates elements set to zero. Regions shown as gray have been multiplied by a nonzero factor as determined by Eq. (7), with $y=10^{-4}$.

representation as

$$
\rho\left(x, x^{\prime}, t\right)=\left\langle x|\hat{\rho}(t)| x^{\prime}\right\rangle=\psi(x, t) \psi^{*}\left(x^{\prime}, t\right) .
$$

The time evolution of the density operator of a nondissipative quantum system is described by the master equation (often also referred to as the quantum Liouville equation)

$$
\frac{\partial \hat{\rho}}{\partial t}=\frac{1}{i \hbar}[\hat{H}, \hat{\rho}],
$$

where $\hat{H}$ is the Hamiltonian operator for the double-well system. We next coarse grain the position into a discrete lattice of position states and expand the system's wave function in a basis of position eigenstates (referred to henceforth as the Pointer state basis): $|\psi(t)\rangle=\sum_{n=1}^{N} C_{n}(t)\left|X_{n}\right\rangle$ so that all quantities are represented on a grid at uniformly spaced points $X_{n}$. Thus, $V_{n} \equiv V\left(X_{n}\right)$ is the value of the potential at grid point $X_{n}$.

Inserting a complete set of states $\sum_{k}\left|X_{k}\right\rangle\left\langle X_{k}\right|$ into each term in the commutator in (2) leads to an equation for the density matrix elements $\rho_{n m}(t)$ of the form

$$
i \hbar \frac{\partial \rho_{n m}}{\partial t}=\sum_{k}\left(H_{n k} \rho_{k m}-\rho_{n k} H_{k m}\right),
$$

where the Hamiltonian matrix elements are

$$
\hat{H}_{n m}=\langle n|H| m\rangle=\left(\frac{-\hbar^{2}}{2 m} \frac{\partial^{2}}{\partial X_{n}^{2}}+V_{n}\right) \delta_{n m} .
$$

For ease of computation we then approximate the second derivative in the kinetic energy operator using the three-point formula

$$
f^{\prime \prime}\left(X_{n}\right) \approx\left[f\left(X_{n-1}\right)-2 f\left(X_{n}\right)+f\left(X_{n+1}\right)\right] / \Delta X^{2},
$$

where $\Delta X$ is the grid spacing. Equation (4) then simplifies through the use of (5) and the fact that the potential is diagonal to

$$
\begin{aligned}
\dot{\rho}_{n m}= & \frac{1}{i \hbar}\left[-\frac{\hbar^{2}}{2 m} \frac{\rho_{n-1, m}+\rho_{n+1, m}-\rho_{n, m-1}-\rho_{n, m+1}}{\Delta X^{2}}\right. \\
& \left.+\left(V_{n}-V_{m}\right) \rho_{n m}\right] .
\end{aligned}
$$

This first-order differential equation in time is solved using Runge-Kutta algorithm for the full $N \times N$ coupled equations (one for each element in the density matrix).

Simulating a Pointer measurement is relatively simple. First, a block size (from anywhere between 1 and $N / 2$, where $N$ is the number of Pointer states) is chosen to determine the precision of the measurement. In this study we choose a block size of $N / 2$, which effectively allows us to know, upon measurement, no more than which side of the barrier the proton is on (an imprecise measurement). At certain chosen intervals, greater than the step size in time in the numerical integration of Eq. (6), we carry out our measurement by multiplying all elements in the off-diagonal blocks of the density matrix by a decay factor

$$
\rho_{n m}=\rho_{n m} e^{-y(n-m)^{2}},
$$

where $y \geqslant 0$ is a harshness parameter. This parameter is necessary to avoid discontinuities in the wave function and its derivative, both of which must be continuous across the block boundaries and therefore so must the elements of the density matrix. A smooth and continuous decay of the matrix elements across block boundaries also makes sense physically in terms of the action of a measurement on the system by its environment. The effect of this is visible in the gradients shown in Fig. 1.

It is clear from Eq. (7) that the closer to the diagonal the element is ( $n-m$ is small) the less affected it is, but for those further away ( $n-m$ is large) the closer to zero the decay factor is, effectively eliminating the matrix element, as shown in Fig. 1.

\section{B. Lindblad method}

The density matrix can also be expanded in the basis of energy eigenstate of the double well, where a smaller matrix is required (since the number of eigenstates needed to describe the wave function is far fewer than the number of grid points considered in the Pointer basis). In this basis the density matrix is

$$
\hat{\rho}=|\psi\rangle\left\langle\psi\left|=\sum_{i j} \alpha_{i} \alpha_{j}^{*}\right| \phi_{i}\right\rangle\left\langle\phi_{j}\right|,
$$

where

$$
\hat{H}\left|\phi_{i}\right\rangle=E_{i}\left|\phi_{i}\right\rangle
$$

which gives a particularly simple form for the time evolution of the density matrix elements

$$
\dot{\rho}_{i j}=\frac{1}{i \hbar}\left(E_{i}-E_{j}\right) \rho_{i j} .
$$


We will discuss later the number of eigenstates necessary in order to achieve good enough accuracy.

In order to model an open (dissipative) system, coupling to the environment (in the limit of weak coupling to a Markovian bath) can be included in the Liouville equation (2), which is generalized to include a dissipative (Lindblad) term on the right-hand side $[48,49]$

$$
\frac{\partial \hat{\rho}}{\partial t}=\frac{1}{i \hbar}[\hat{H}, \hat{\rho}]+\hat{L} \hat{\rho},
$$

where this extra term generally takes the form

$$
\hat{L} \hat{\rho}=\sum_{\beta}\left(\hat{A}_{\beta} \hat{\rho} \hat{A}_{\beta}^{\dagger}-\frac{1}{2}\left[\hat{A}_{\beta}^{\dagger} \hat{A}_{\beta}, \hat{\rho}\right]_{+}\right)
$$

and the operators $\hat{A}_{\beta}$ are defined as [50]

$$
\hat{A}_{\beta}=\sqrt{W_{i j}}|i\rangle\langle j| \text {. }
$$

The index $\beta$ labels ordered pairs $(i, j)$ of energy eigenstates and $W_{i j}$ are environment-induced transition rates between well states $|i\rangle$ and $|j\rangle$. This leads to

$$
\hat{L} \hat{\rho}=\sum_{i j} W_{i j}\left(|i\rangle\langle j|\hat{\rho}| j\rangle\langle i|-\frac{1}{2}[|j\rangle\langle j|, \hat{\rho}]_{+}\right) .
$$

Substituting the above back into Eq. (11) leads to diagonal and off-diagonal density matrix elements in the eigenstate basis

$$
\begin{aligned}
& \dot{\rho}_{i j}=\frac{1}{i \hbar}\left(E_{i}-E_{j}\right) \rho_{i j}-\frac{1}{2} \rho_{i j} \sum_{k}\left(W_{k i}+W_{k j}\right), \quad i \neq j, \\
& \dot{\rho}_{i i}=\sum_{k}\left(W_{i k} \rho_{k k}\right)-\rho_{i i} \sum_{k}\left(W_{k i}\right) .
\end{aligned}
$$

There are a number of ways of calculating the transition (relaxation) matrix elements $W_{i j}$. They are derived here using the microscopic theory of Meyer and Ernst [5]. We will not repeat the details of the derivation, but will summarize the assumptions and inputs to the model briefly. The full system plus bath Hamiltonian is written as

$$
\hat{H}=\hat{H}_{s}+\hat{H}_{b}+\Delta \hat{H},
$$

where $\hat{H}_{s}$ is the system Hamiltonian involving a kinetic energy operator for the tunneling particle along with the double-well potential and $\hat{H}_{b}$ is the bath Hamiltonian defined as a sum of harmonic oscillators

$$
\hat{H}_{b}=\frac{1}{2} \sum_{m}\left(p_{m}^{2}+\omega_{m}^{2} q_{m}^{2}\right),
$$

where $m$ is the set of bath oscillators, $p_{m}$ are their momenta, $q_{m}$ are their spatial positions, and $\omega_{m}$ are their frequency. Finally, $\Delta \hat{H}=\zeta \sum_{m} f_{m} q_{m}$ is the interaction between the system and bath, with coupling constant $f_{m}$.

The transition probability $W_{i j}$ between states $i$ and $j$ is defined as

$$
\begin{gathered}
W_{i j}=\frac{1}{\hbar^{2}} \int_{-\infty}^{\infty} d \tau e^{-i \omega_{i j} \tau} C_{i j}(\tau), \\
W_{j j}=-\sum_{i \neq j} W_{i j},
\end{gathered}
$$

where $\omega_{i j}$ is a transition frequency depending on the energy of the eigenstates $i$ and $j$,

$$
\omega_{i j}=\frac{E_{i}-E_{j}}{\hbar},
$$

and the transition probabilities $W_{i j}$ according to this definition will automatically fulfill the principle of detailed balance [5].

The correlation functions $C_{i j}$ required in Eq. (18) are calculated from an appropriately chosen power spectral density function of the active bath displacement

$$
J_{r r}(\omega)=\frac{4 \sqrt{2} \Delta V_{R} \hbar \omega_{p} \omega^{3}}{\left(\omega_{p}^{4}+\omega^{4}\right)\left(e^{\hbar \omega / k_{B} T}-1\right)},
$$

where $T$ is temperature, $\omega_{p}$ is a characteristic phonon frequency of the heat bath, and $\Delta V_{R}$ is the rearrangement energy gained by the bath oscillators upon displacement from $q_{m}=0$ to their optimal values at the potential minima [5]. This definition of the power spectral density function is related to the chosen model for the bath oscillators, using Debye theory, whereby the product of the square of the coupling constants and the density of modes increases with $\omega^{4}$ at low frequencies and becomes constant at $\omega_{p}$.

\section{NUMERICAL RESULTS}

We use an analytic one-dimensional asymmetric doublewell potential with a quartic dependence on position [5]

$$
V(\zeta)=\left[B\left(1-\zeta^{2}\right)\right]^{2}+\frac{\Delta V}{2} \zeta,
$$

where $B$ is the barrier height, $\Delta V$ is the asymmetry parameter, and the dimensionless variable $\zeta$ is a reduced proton position transfer coordinate. The parameters were chosen, as in [5], to describe the benzoic acid dimer: $B=620 \mathrm{~cm}^{-1}$ and $\Delta V=63.6 \mathrm{~cm}^{-1}$. Figure 2 shows the chemical structure of the benzoic acid dimer, with its double $\mathrm{H}$ bond, and the shape of the resulting double-well potential. Also plotted are the first six eigenfunctions and their corresponding energies, calculated from a numerical solution of the Schrödingerequation using the a Runge-Kutta routine.

As a test of the two approaches, we first carry out calculations for the isolated system, that is, without setting the off-diagonal elements of the density matrix to zero in the Pointer method and without the Lindblad term in the eigenstate basis (achieved by setting the temperature $T=0$ in the spectral function $J$ ). An initial Gaussian wave function, centered at $\zeta=-1$, was chosen to represent a proton that starts off at $t=0$ in the deeper well. The density matrix was then evolved in time in each case and the probability of finding the proton in the shallow well was calculated at appropriate time steps. Due to the asymmetry of the double well, the ground-state eigenfunction exists mainly in the deeper well and the initial Gaussian wave function consists almost entirely of this lowest eigenfunction. Crucially of course, it will contain small components of higher eigenstates and is thus not a stationary state. A small component of the wave function will therefore tunnel through to the shallow well.

In the absence of any interaction with the environment, the numerical accuracy of the two methods can be tested against each other to determine the sizes of the basis sets required 


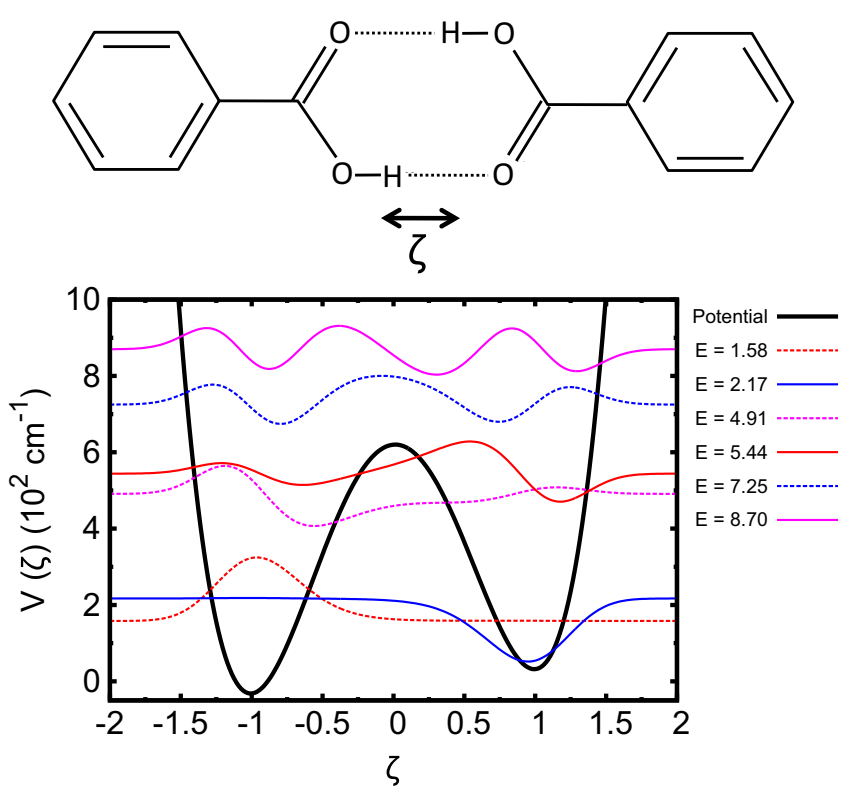

FIG. 2. (Color online) Double-well potential for hydrogen transfer in benzoic acid dimer, showing the first six energy eigenstates plotted at their appropriate energies on the $y$ axis. States 1 and 2 are wholly localized in their respective wells and states 3 and 4 are mostly localized in their respective wells.

for sufficient accuracy. It was found that the eigenstate basis required 16 terms, which produces results matching those obtained in the Pointer state basis to within an accuracy of one part in $10^{5}$ over the whole time period of interest. Although rather difficult to see, there are in fact two curves in Fig. 3. The low probability of finding the proton in the shallow well reflects the choice of initial wave function and we stress the agreement between the two approaches is no more than a test of the numerical calculations.

It is important to add that the many fluctuations seen in this figure are not indicative of individual proton-tunneling

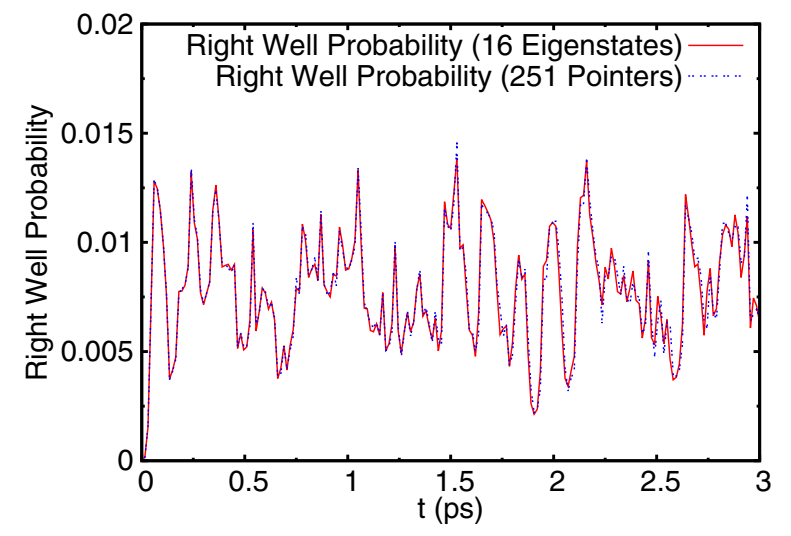

FIG. 3. (Color online) Probability of a proton being in the shallow well over a period of 3 ps using optimum basis sizes with no measurement or environmental coupling. The results match extremely well, demonstrating the accuracy of the two quite different approaches. events, but are due to the complex physical interference effects resulting from the time dependence of the part of the wave function in the shallow well. As such, this figure is no more than a test that both numerical procedures are describing the same physics. We have investigated the time scales for tunneling to take place and find that, for the case of a symmetric, or very near symmetric, double well, the tunneling time is in the range of $10-20$ ps. (Note that, in the absence of measurement we cannot obtain a tunneling time using our asymmetric well because it is only the action of the environment in exciting the initial state that triggers any measurable tunneling.)

We next include the coupling to the environment by (a) adding the dissipative Lindblad term in the eigenstate basis and (b) carrying out Pointer measurements at regular intervals. Instead of taking a Gaussian centered in the deeper well for the initial wave function (depicting a proton that is definitely in the deeper well to begin with), we now choose the more realistic case of the ground-state eigenfunction $|\psi(t=0)\rangle=\left|\phi_{0}\right\rangle$. Due to the asymmetry of the well, this eigenfunction is almost entirely in the deep well anyway and closely resembles the Gaussian shape. However, this is a stationary state and any tunneling will now be due entirely to the coupling of the system to the environment. In the Lindblad method, this is due to the external heat bath inducing transitions between the ground state and higher-energy eigenstates that have larger components in the shallow side of the well (which is just another way of saying it enhances the tunneling probability). Since this coupling depends on the temperature of the bath we should expect an enhanced tunneling probability with higher temperature (thermally assisted tunneling). In contrast, the interpretation in the Pointer method is that repeated measurement (setting the off-diagonals of the density matrix to zero) provides an unavoidable kick to the proton pushing it to higher-energy states.

Clearly, if the coupling is strong enough in both pictures the proton could be induced into hopping over the barrier classically rather than tunneling through it. To test this, we compared two eigenstate basis calculations with basis sizes of 4 and 16 eigenstates, respectively. The first four energy eigenvalues lie below the top of the barrier and so, with a basis that only includes coupling between these states, quantum tunneling would be the only way for the proton to find itself on the other side of the barrier. On the other hand, including an additional 12 states would allow classical over the barrier hopping. Hardly any difference at all was found between the two cases, implying that tunneling is the dominant mechanism in this case. In addition, the occupation probability of each eigenstate (obtained by finding the overlap between the wave function and each eigenstate) over time was checked for the two cases of basis size.

Figure 4 shows how the occupation probability for first four eigenstates changes over time. When using the larger basis, the 12 higher-energy eigenstates have a cumulative occupation probability of $6 \%$ at 20 ps. What is clear is that adding the dissipative Lindblad term allows primarily for strong transitions between the ground state and the first excited state (which is predominantly in the shallow well). Thus, the thermally assisted tunneling is almost entirely due to populating this state. 


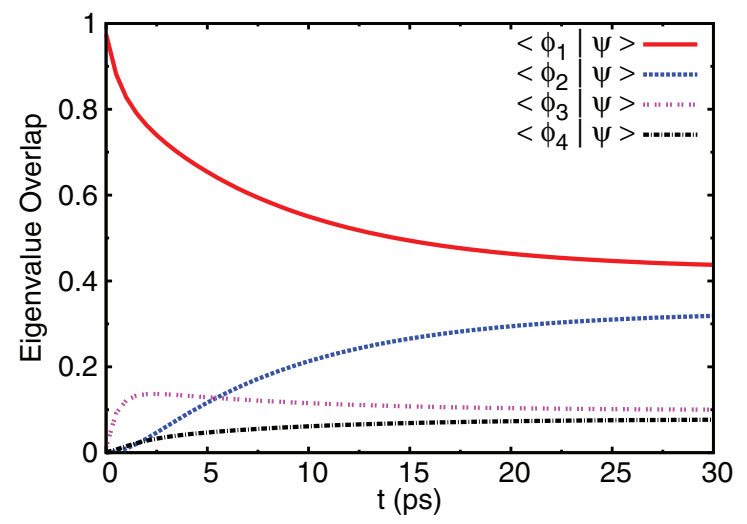

FIG. 4. (Color online) Overlap of the wave function with each of the first four energy eigenstates over a period of $20 \mathrm{ps}$.

Figure 5 shows a comparison between two sets of curves. The solid lines are from the eigenstate basis calculation at three different bath temperatures $(115,155$, and $200 \mathrm{~K})$. Overlaying these are the results (dashed lines) from the Pointer calculation with measurements being made at three different frequencies of 20,100 , and $3300 \mathrm{ps}^{-1}$. (Note the numerical step size in $t$ is $0.05 \mathrm{fs}$.) It is clear from this graph that increasing the temperature of the bath leads to stronger coupling to the environment and hence enhanced rate of thermally assisted tunneling. This is very strongly and neatly correlated with a similar enhancement in tunneling probability with increased frequency of observation and measurement, what might be referred to as an anti-Zeno effect. Making the link in this way with the dissipative Lindblad approach clarifies why this is so.

Figures 6 and 7 consider two different snapshots in time, at $t=10$ and $100 \mathrm{ps,}$ and compares the probability of the proton having tunneled as a function of temperature in the Lindblad approach with its probability of having tunneled as a function of observation frequency in the Pointer approach. A similar picture is seen at both times: Increasing the frequency of measurement by the environment is equivalent to raising its

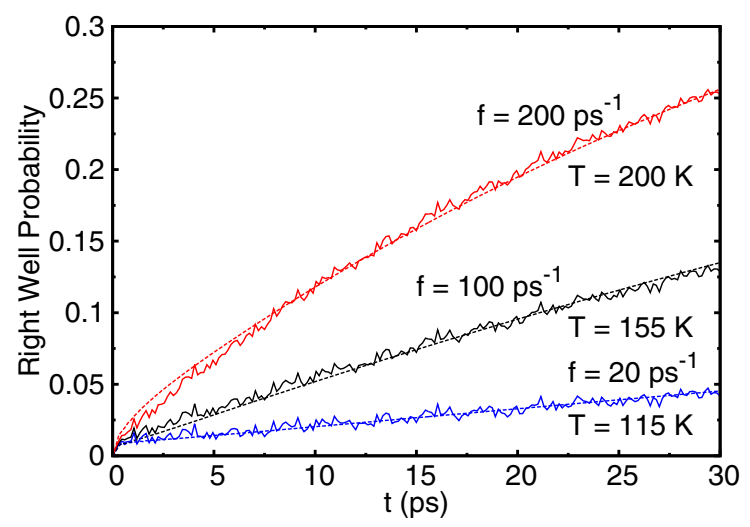

FIG. 5. (Color online) Probability of the proton being in the shallow well during a period of $30 \mathrm{ps}$ using Pointer state measurements with varying observation frequencies $f$ and the Lindblad term with varying temperatures $T$. Observation size is fixed at $0.5 \mathrm{~N}$ and harshness is set at $10^{-4}$.

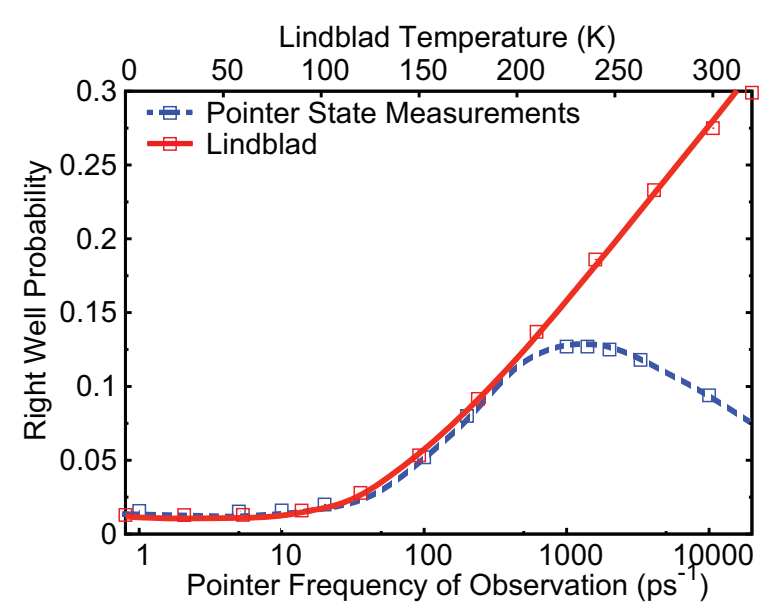

FIG. 6. (Color online) Probability of the proton being in the shallow well after 10 ps using Pointer state measurements with varying observation frequencies and the Lindblad approach with varying temperatures. The sold curves have been drawn through the discrete calculated points to guide the eye.

temperature; both lead to enhanced tunneling probability, or anti-Zeno effect.

Beyond $200 \mathrm{~K}$ in the Lindblad approach, the tunneling probability continues to rise until it reaches a maximum of $P \leqslant 0.5$, depending on the asymmetry in the double well. Figure 4 explains the reason for this leveling off since, after about $20 \mathrm{ps}$, the occupation probabilities of each energy eigenstate remain constant. On the other hand, increasing the measurement frequency in the Pointer model is equivalent to imparting a kick to the proton, exciting to higher-energy states where it can tunnel more easily. However, when the measurements are made too frequently (beyond $1000 \mathrm{ps}^{-1}$ ) we see a drop in tunneling probability and a clear change from the anti-Zeno to the Zeno effect. This can be interpreted as the wave function being collapsed back to its initial state (by

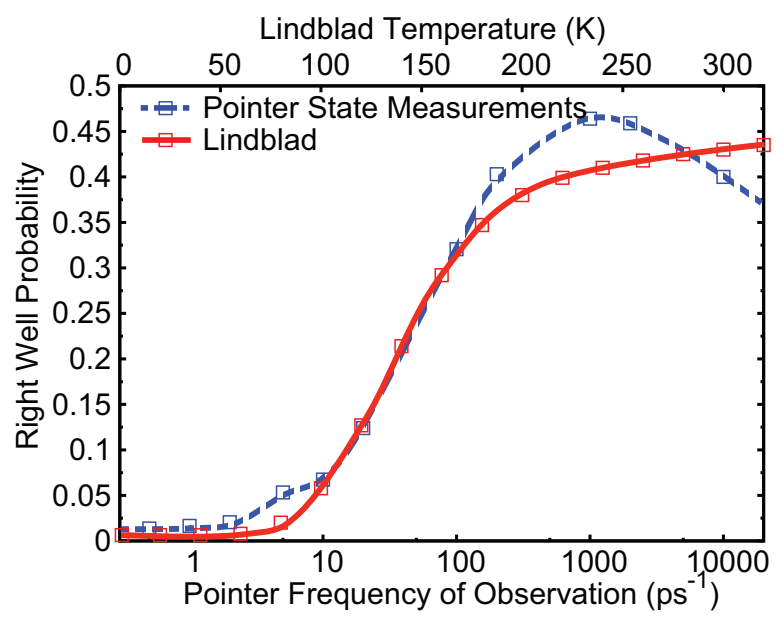

FIG. 7. (Color online) Probability of the proton being in the shallow well after 100 ps using Pointer measurements with varying observation frequencies and the Lindblad approach with varying temperatures. The solid curves have been drawn through the discrete calculated points to guide the eye. 
setting the off-diagonals of the density matrix to zero) so often that it does not have as much chance to evolve and the proton is less likely to tunnel.

\section{DISCUSSION}

We have modeled proton tunneling in an asymmetrical double-well potential such as that which can be found in many chemical and biological systems, including the benzoic acid dimer considered here, and simulated the way the system evolves in time by calculating the probability of finding the proton on the opposite side of the potential barrier. For an isolated quantum system and our chosen potential parameters, the proton is very unlikely to tunnel across into the shallow well since the initial wave function is almost entirely in the deep well. However, when the system is allowed to couple to an external environment its evolution is altered dramatically. We have considered two very different pictures of the way this environmental coupling takes place and have shown that within a certain domain there is a clear correspondence between the two.

More specifically, at any given temperature of the surrounding heat bath, but below a certain upper value, and allowing the combined system of well plus bath to evolve in time, one can always find a corresponding frequency of von Neumann-type observation (collapse of the wave function) that mimics the same evolution in time of the tunneling probability. For example, by starting the proton in the deeper well at $t=0$, coupling to a bath at some temperature, and then allowing it to evolve in time, we show that the probability of tunneling gradually increases over time. This well-known behavior is due the interaction with the bath leading to transitions between energy eigenstates and hence contributions to the proton wave function from higher states, and a correspondingly significant and growing amplitude in the shallow well. Physically, we can think of the proton as populating higher-energy states closer to the top of the potential barrier. In principle, these could even include states above the barrier, allowing for the proton to get across via over the barrier classical hopping. However, we find that for our chosen potential well geometry, the transition probabilities to energies above the barrier are too small to contribute. Therefore, the enhanced probability of finding the proton in the shallow well is indeed due almost entirely to tunneling.

When we consider the quite different picture of simulating decoherence by setting the off-diagonal elements of the density matrix to zero at regular intervals (with a well-defined frequency) we see the same increase over time in the tunneling probability. For example, the behavior of the system coupled to a heat bath at $155 \mathrm{~K}$ looks like one in which the off-diagonal elements of the density matrix are collapsed every 0.01 ps. Of course, the absolute numerical values stated here are model dependent and therefore only qualitative conclusions should be drawn from these results.

This mapping suggests that despite the two quite different models of decoherence, they must be describing the same physical process. In the Pointer approach, rather than using the language of transition probabilities between energy eigenstates, we can think of frequent measurements as disturbing the system: exciting the proton by giving it a kick to higherenergy levels where tunneling is easier. This correspondence suggests that quantum measurements may effectively drive a system to a given virtual temperature: The more frequent the measurements, the higher the virtual temperature of the environment carrying out these measurements.

However, this mapping only works up to a point. Above a certain temperature and frequency of measurement, the correspondence between the two pictures breaks down. In contrast, increasing the bath temperature further continues to enhance the tunneling probability, albeit at a slower rate as it reaches saturation: A maximum tunneling probability that depends on the geometry of the well, increasing the measurement frequency further in the Pointer method, causes a reversal in behavior and the tunneling probability begins to fall due to the quantum Zeno effect. Thus, rather than the proton being given ever more frequent kicks to increase its energy and enhance the tunneling probability, now a competing mechanism starts to dominate as the wave function is being collapsed so frequently that it does not have enough time to evolve.

In summary, at least for the simple model described here, increasing the strength of coupling to the environment (achieved by raising the temperature of the heat bath) leads to a clear anti-Zeno effect of enhancing the tunneling rate: thermally assisted tunneling. This rate continues to rise over time, reaching a maximum that depends on the asymmetry of the well. Up to a certain temperature this enhanced tunneling can be mimicked very well by increasing the frequency of a von Neumann-type measurement. However, increasing the frequency of measurement further leads to a changeover from the anti-Zeno to the Zeno effect, whereby the tunneling rate starts to decrease again. However, in the more physically realistic Lindblad approach of continuous coupling to an external heat bath no such Zeno effect is seen, even at high temperatures. We conclude therefore that environmentally induced decoherence for the case of proton tunneling leads entirely to a quantum anti-Zeno effect.

\section{ACKNOWLEDGMENT}

The authors would like to thank the Engineering and Physical Sciences Research Council for funding the Doctoral Training Centre and this work.
[1] W. H. Zurek, Phys. Today 44(10), 36 (1991).

[2] W. H. Zurek, Rev. Mod. Phys. 75, 715 (2003).

[3] A. Oppenlander, C. Rambaud, H. P. Trommsdorff, and J.-C. Vial, Phys. Rev. Lett. 63, 1432 (1989).
[4] A. Heuer and U. Haeberlen, J. Chem. Phys. 95, 4201 (1991).

[5] R. Meyer and R. R. Ernst, J. Chem. Phys. 93, 5518 (1990).

[6] J. L. Skinner and H. P. Trommsdorff, J. Chem. Phys. 89, 897 (1988). 
[7] A. J. Horsewill, P. J. McDonald, and D. Vijayaraghavan, J. Chem. Phys. 100, 1889 (1994).

[8] D. F. Brougham, A. J. Horsewill, and R. I. Jenkinson, Chem. Phys. Lett. 272, 69 (1997).

[9] D. F. Brougham, A. J. Horsewill, and H. P. Trommsdorff, Chem. Phys. 243, 189 (1999).

[10] A. J. Horsewill, D. F. Brougham, R. I. Jenkinson, C. J. Mcgloin, H. P. Trommsdorff, and M. R. Johnson, Ber. Bunsen. Phys. Chem. 102, 317 (1998).

[11] M. A. Neumann, S. Craciun, A. Corval, M. R. Johnson, A. J. Horsewill, V. A. Benderskii, and H. P. Trommsdorff, Ber. Bunsen. Phys. Chem. 102, 325 (1998).

[12] M. Neumann, D. F. Brougham, C. J. McGloin, M. R. Johnson, A. J. Horsewill, and H. P. Trommsdorff, J. Chem. Phys. 109, 7300 (1998).

[13] A. J. Horsewill, C. J. McGloin, H. P. Trommsdorff, and M. R. Johnson, Chem. Phys. 291, 41 (2003).

[14] Y. Cha, C. J. Murray, and J. P. Klinman, Science 243, 1325 (1989).

[15] M. H. Glickman, J. S. Wiseman, and J. P. Klinman, J. Am. Chem. Soc. 116, 793 (1994).

[16] L. Masgrau, A. Roujeinikova, L. O. Johannissen, P. Hothi, J. Basran, K. E. Ranaghan, A. J. Mulholland, M. J. Sutcliffe, N. S. Scrutton, and D. Leys, Science 312, 237 (2006).

[17] A. Kohen, R. Cannio, S. Bartolucci, J. P. Klinman, and J. P. Klinman, Nature (London) 399, 496 (1999).

[18] N. S. Scrutton, J. Basran, and M. J. Sutcliffe, Eur. J. Biochem. 264, 666 (1999).

[19] H. Fearn and W. E. Lamb, Jr., Phys. Rev. A 46, 1199 (1992).

[20] T. P. Altenmuller and A. Schenzle, Phys. Rev. A 49, 2016 (1994).

[21] B. Kaulakys and V. Gontis, Phys. Rev. A 56, 1131 (1997).

[22] K. Koshino and A. Shimizu, Phys. Rep. 412, 191 (2005).

[23] A. G. Kofman and G. Kurizki, Nature (London) 405, 546 (2000).

[24] J. Ruseckas and B. Kaulakys, Phys. Rev. A 63, 062103 (2001).

[25] J. Ruseckas and B. Kaulakys, Phys. Rev. A 69, 032104 (2004).
[26] J. von Neumann, Mathematical Foundations of Quantum Mechanics (Princeton University Press, Princeton, 1955).

[27] P. E. Parris and R. Silbey, J. Chem. Phys. 83, 5619 (1985).

[28] A. I. Larkin and Y. N. Ovchinnikov, Zh. Eksp. Teor. Fiz. 91, 325 (1986).

[29] P. Hänggi, P. Talkner, and M. Borkovec, Rev. Mod. Phys. 62, 251 (1990).

[30] H. Dekker, Phys. Rev. A 44, 2314 (1991).

[31] H. Dekker, Physica A 175, 485 (1991).

[32] H. Dekker, Physica A 176, 220 (1991).

[33] H. Dekker, Physica A 178, 289 (1991).

[34] H. Dekker, Physica A 179, 81 (1991).

[35] H. Dekker, Physica A 210, 507 (1994).

[36] T. Dittrich, B. Oelschlägel, and P. Hänggi, Europhys. Lett. 22, 5 (1993).

[37] M. Topaler and N. Makri, J. Chem. Phys. 101, 7500 (1994).

[38] D. R. Reichman and R. J. Silbey, J. Phys. Chem. 99, 2777 (1995).

[39] R. I. Cukier, M. Morillo, K. Chun, and N. O. Birge, Phys. Rev. B 51, 13767 (1995).

[40] D. A. Garanin and E. M. Chudnovsky, Phys. Rev. B 56, 11102 (1997).

[41] M. Grifoni and P. Hänggi, Phys. Rep. 304, 229 (1998).

[42] S. Kohler, R. Utermann, P. Hänggi, and T. Dittrich, Phys. Rev. E 58, 7219 (1998).

[43] M. N. Leuenberger and D. Loss, Phys. Rev. B 61, 1286 (2000).

[44] A. J. Horsewill, Prog. Nucl. Magn. Reson. Spectrosc. 52, 170 (2008).

[45] D. Wallace, Phys. Rev. A 63, 022109 (2001).

[46] J. S. Al-Khalili and P. D. Stevenson, Adv. Sci. Lett. 1, 140 (2008).

[47] B. Misra and E. C. G. Sudarshan, J. Math. Phys. 18, 756 (1977).

[48] V. Gorini, A. Kossakowski, and E. C. G. Sudarshan, J. Math. Phys. 17, 821 (1976).

[49] G. Lindblad, Commun. Math. Phys. 48, 119 (1976).

[50] C. Scheurer and P. Saalfrank, J. Chem. Phys. 104, 2869 (1996). 